\title{
Refinement of Mouse Protocols for the Study of Platelet Thromboembolic Responses In Vivo
}

\author{
Francesca Rauzi $^{1} \quad$ Erica Smyth $^{1}$ Michael Emerson ${ }^{1}$ \\ ${ }^{1}$ Section of Molecular Medicine, National Heart and Lung Institute, \\ Imperial College London, London, United Kingdom \\ Thromb Haemost 2017;117:2283-2290.

\begin{abstract}
Address for correspondence Michael Emerson, PhD, Section of Molecular Medicine, National Heart and Lung Institute, Imperial College London, Sir Alexander Fleming Building, Exhibition Road, London SW7 2AZ, United Kingdom

(e-mail: m.emerson@imperial.ac.uk).
\end{abstract}

\begin{abstract}
Keywords

- mouse model

- platelets

- thromboembolism

Mouse models of thromboembolism are frequently used to investigate platelet function in vivo and, according to European Union (EU) legislation, must be conducted in the context of replacement, refinement and reduction. We have previously developed a refined real-time mouse model of thromboembolism as an alternative to models of thromboembolic mortality which inflict considerable pain and suffering. Real-time monitoring involves infusion of radiolabelled platelets into the circulation of anaesthetized mice, and platelet aggregation is measured as increases in platelet-associated counts in the pulmonary vasculature following injection of platelet agonists. This gives a definitive data set on the tissue localization and extent of platelet activation. We developed an additional, more simplistic alternative to mortality models based on blood microsampling which entails the measurement of circulating platelet counts following agonist stimulation. Blood microsamples were collected from the tail vein of anaesthetized mice at three different time points leading to a reduction in animal numbers. Platelet counts significantly dropped 1 minute after stimulation with collagen or thrombin and were restored over 10 minutes. These results correlate with those obtained via real-time monitoring and were confirmed by immunohistochemistry. Pre-treatment of mice with aspirin significantly inhibited the decrease in platelet counts following collagen. These data suggest that blood microsampling may be implemented as a simplistic refined alternative to mortality models of thromboembolism when specialized monitoring equipment, or use of radioactive isotopes for realtime monitoring, which remains the 'gold standard', is not feasible. Microsampling refines and reduces animal procedures in compliance with current EU legislation.
\end{abstract}

\section{Introduction}

Animal models allow the investigation of platelets in the contexts of haemostasis, thrombosis and inflammation while taking into account systemic factors, including the vascular endothelium, which are not present in isolated platelet preparations. $^{1-3}$ The genetic malleability of the mouse makes it the species of choice for many biomedical researchers. Research involving the use of animals within the European Union (EU) is regulated by a directive requiring explicit application of replacement (avoidance of animal use), reduction (use of fewer animals) and refinement (modifying procedures to reduce severity) of animal procedures (the 3Rs).

Thromboembolic mortality models in which platelet agonists such as collagen, thrombin and adenosine diphosphate (ADP) are injected into the tail vein of conscious mice are currently used by significant numbers of research groups to assess platelet function in vivo. ${ }^{4-6}$ These models employ death or hind limb paralysis as endpoints, induce extensive pain and suffering since anaesthesia is often not used and involve large received

April 10, 2017

accepted after revision

August 16, 2017
DOI https://doi.org/

10.1160/TH17-04-0250.

ISSN $0340-6245$.
Copyright () 2017 Schattauer

License terms

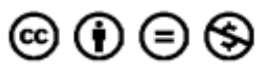


numbers of animals ( $\mathbf{- T a b l e} \mathbf{1}$ ). The scientific value of these models has also been questioned. ${ }^{7}$

Our group has previously developed a refined real-time mouse model of thromboembolism as an alternative to thromboembolic mortality models. ${ }^{8}$ In this model, platelet aggregation is measured directly and in situ in mice under terminal anaesthesia. The model consists of monitoring ${ }^{111}$ In-radiolabelled platelets that freely circulate and accumulate in the pulmonary vascular bed following platelet activation with non-lethal doses of platelet agonists. In vivo platelet aggregation is measured as increases in platelet-associated counts via external scintillation probes above the pulmonary region, thus providing the important data set of quantitative distribution. ${ }^{8,9}$ The use of general anaesthesia means that painful procedures are avoided and, unlike mortality models, observations may be directly attributed to platelets.

Real-time radiolabelled platelet monitoring has been shown to be sensitive enough to identify environmental or nutritional drivers of cardiovascular risk or protection. For example, our group has shown that platelet aggregation in vivo is enhanced by diesel exhaust particles or engineered nanoparticles and inhibited by inorganic nitrate/nitrite. ${ }^{10-12}$ When applied, the model has led to up to $85 \%$ reduction in mouse use compared with comparable studies using thromboembolic mortality models. $7,13,14$

Platelet monitoring in vivo requires particular technical skills and handling of radioactive isotopes along with extensive facilities and equipment. These factors have limited implementation by groups currently using mortality models of thromboembolism. We therefore sought novel and refined approaches to study platelet aggregation in vivo that could be more easily adopted. We now report a non-invasive and more simplistic approach to study platelet function in vivo based on blood microsampling, with the caveat that the extent of platelet localization obtained by platelet monitoring in vivo is lacking. Moreover, since indium oxine is no longer easily available in many parts of Europe, including the
United Kingdom, we characterized indium tropolone for use in real-time monitoring.

\section{Methods}

\section{Mice}

Balb/c mice (20-25 g) were purchased from Harlan (Bicester, UK) and had access to food and water ad libitum. All procedures were conducted under our Home Office Project License PPL 70/8791 and were approved by the Ethical Review Panel at Imperial College London, United Kingdom. Protocols were refined in association with the National Centre for the Replacement, Refinement \& Reduction of Animals in Research (NC3Rs) ${ }^{8}$ and are reported in accordance with the ARRIVE (Animal Research: Reporting of In Vivo Experiments) guidelines for reporting experiments involving animals. ${ }^{15}$

\section{Materials}

Reagents were purchased from Sigma (Dorset, UK) with the exception of indium oxine (GE Healthcare, Bucks, UK), indium chloride (Mallinckrodt Pharmaceuticals, Institute of Nuclear Medicine UCLH, London UK), tropolone (Alfa Aesar, Heysham, United Kingdom) and collagen (Nycomed Pharma, Unterschleissheim, Germany).

\section{Blood Collection and Platelet Labelling with Indium Oxine or Indium Tropolone}

Blood $(0.7-1.0 \mathrm{~mL})$ was collected from terminally anaesthetized donor mice (urethane $25 \% \mathrm{w} / \mathrm{v} ; 10 \mu \mathrm{L} / \mathrm{g}$ ) by cardiac puncture into acidified citrate solution (acid citrate-dextrose (ACD); $200 \mu \mathrm{L}) . \mathrm{A} \mathrm{Ca}^{2+}$ - and $\mathrm{Mg}^{2+}$-free Tyrode's buffer was prepared (CFTS: $138-\mathrm{mM} \mathrm{NaCl}, 2.6-\mathrm{mM} \mathrm{KCl}, 5.5-\mathrm{mM}$ glucose, $12-\mathrm{mM} \mathrm{NaHCO}_{3}$ and $\left.0.2-\mathrm{mM} \mathrm{NaHPO}_{4}\right)$ and used to prepare a CFTS/ACD solution (10:1 mixture) with $0.34 \mu \mathrm{L} / \mathrm{mL} \mathrm{PGE}_{1}$ (stock $1 \mathrm{mg} / \mathrm{mL}$ ). Blood was centrifuged ( 3 minutes; $300 \times g$ ) to obtain platelet-rich plasma (PRP). CFTS/ACD solution

Table 1 List of publications using mouse mortality models of thromboembolism in 2016

\begin{tabular}{|c|c|c|c|c|c|c|}
\hline $\begin{array}{l}\text { Research groups } \\
\text { (2016) }\end{array}$ & $\begin{array}{l}\text { Number of } \\
\text { animals/group }\end{array}$ & $\begin{array}{l}\text { Total number } \\
\text { of animals }\end{array}$ & $\begin{array}{l}\text { Duration of } \\
\text { procedure }\end{array}$ & Platelet agonist & Anaesthesia & Endpoints \\
\hline Assafim 22 & Not reported & Not reported & $15 \mathrm{~min}$ & Thrombin & No & Death \\
\hline $\mathrm{Choi}^{23}$ & 10 & 50 & $15 \mathrm{~min}$ & Thrombin & No & Paralysis/death \\
\hline Huang $^{24}$ & 5 & 15 & $1 \mathrm{~h}$ & Collagen/adrenaline & Yes & Death \\
\hline $\mathrm{Kim}^{25}$ & 15 & 75 & $15 \mathrm{~min}$ & Collagen/adrenaline & No & Death \\
\hline Park $^{26}$ & 40 & 160 & $15 \mathrm{~min}$ & Collagen/adrenaline & No & Paralysis/death \\
\hline Misra $^{27}$ & $\begin{array}{l}5 \text { (control) } \\
10 \text { (treatment) }\end{array}$ & 25 & $15 \mathrm{~min}$ & Collagen/adrenaline & No & Paralysis/death \\
\hline Saito $^{28}$ & 3 & 15 & 5 min to 5 days & Thrombin & No & Death \\
\hline $\operatorname{Lee}^{29}$ & 20 & Not reported & $15 \mathrm{~min}$ & Collagen/adrenaline & No & Paralysis/death \\
\hline $\mathrm{Kim}^{30}$ & $\begin{array}{l}10 \text { (control) } \\
16 \text { (treatment) }\end{array}$ & 58 & $15 \mathrm{~min}$ & Collagen/adrenaline & No & Paralysis/death \\
\hline
\end{tabular}

Note: The table reports a PubMed search list of publications using mortality models of thromboembolism in 2016 together with the number of animals (total and per group), platelet agonist, anaesthetic and endpoints used. PubMed keywords: animal/mouse model thromboembolism, animal/mouse model thrombosis. 
(400 $\mu \mathrm{L}$ ) was added to the remaining red blood cells (RBCs) and centrifuged again ( 3 minutes; $300 \times$ g) to acquire additional PRP. The PRP obtained was spun ( 2 minutes; $200 \times g$ ) to remove contaminating RBCs. Platelets were pelleted ( 7 minutes; $1,500 \times \mathrm{g}$ ) and pooled together by carefully re-suspending them in $1 \mathrm{~mL}$ of CFTS/ACD. Pooled platelets were incubated with $1.8 \mathrm{MBq}{ }^{111}$ In oxine or ${ }^{111}$ in tropolone at room temperature for 10 minutes (indium chloride and tropolone $1 \mu \mathrm{g} / \mu \mathrm{L}$ were pre-incubated for 10 minutes prior to labelling platelets). Radioactive counts were measured in samples at this point. Platelets were pelleted (5 minutes; $1,500 \times g$ ) and the radioactive supernatant discarded. The surface of the pellet was carefully washed with CFTS to remove excess radiation and the pellet was then gently resuspended in CFTS in a volume of approximately $250 \mu \mathrm{L}$ per mouse. Radioactive counts were measured again to calculate percentage labelling efficiency in five independent preparations. Platelets were left resting for 30 minutes to allow inhibitors to dissipate.

\section{Real-Time Platelet Monitoring In Vivo}

Radiolabelled platelets were infused via an exposed femoral vein into anaesthetized Balb/c mice (urethane 25\% w/v; 10 $\mu \mathrm{L} / \mathrm{g}$ ) and allowed to equilibrate for 20 minutes as previously described. ${ }^{8}$ Platelet aggregation was induced through the intravenous injection of collagen $(50 \mu \mathrm{g} / \mathrm{kg})$ and platelet responses were measured as increases in platelet-associated radioactive counts in the pulmonary vascular bed. Data were acquired with a Single Point Extended Area Ratio (SPEAR) probe (eV products, Saxonburg, Pennsylvania, United States) positioned over the pulmonary vasculature and custom software (Mumed systems, London, United Kingdom) as previously described. ${ }^{8}$

\section{Blood Sampling via Cardiac Puncture}

Anaesthetized (urethane 25\% w/v, $10 \mu \mathrm{L} / \mathrm{g}$ ) Balb/c mice were injected intravenously via the femoral vein with increasing doses of collagen $(12.5-50 \mu \mathrm{g} / \mathrm{kg}$ ) or saline. Blood was collected by cardiac puncture 1 minute after agonist injection and incubated with Stromatol (1:100 DF) to measure platelet counts with a haemocytometer (platelets $/ \mathrm{mL}$ ).

\section{Blood Microsampling}

Balb/c mice were pre-warmed $\left(37^{\circ} \mathrm{C}\right)$ in a surgery recovery box for 10 minutes to facilitate both microsampling and injections. Animals were anaesthetized with isoflurane ( 2 minutes) in the induction box and then transferred to the mask/set-up (-Supplementary Figure S1A [online only]), maintained under general anaesthesia and ultimately overdosed with pentobarbital. A small incision was performed with a blade on the distal portion of the tail vein (-Supplementary Figure S1B [online only]) and a blood microsample $(2 \mu \mathrm{L})$ was collected with a pipette $(10 \mu \mathrm{L})$, transferred into an Eppendorf tube containing Stromatol (1:100 DF) and mixed to measure platelet counts (platelets $/ \mathrm{mL}$ ). Bleeding was promptly stopped by putting pressure on the site of the incision with tissue immediately after blood collection. Collagen $(50 \mu \mathrm{g} /$ $\mathrm{kg})$, thrombin (65 IU $/ \mathrm{kg}$ ) or ADP $(400 \mu \mathrm{g} / \mathrm{kg}$ ) were then injected intravenously in $100 \mu \mathrm{L}$ volumes via the same tail vein above the incision (-Supplementary Figure S1C [online only] and https://vimeo.com/192527541). Additional small incisions were performed with a blade 1 and 10 minutes after platelet activation on the unused tail vein and blood samples $(2 \mu \mathrm{L})$ collected to measure platelet counts ( - Supplementary Figure S1D [online only]). In aspirin studies, mice were injected intraperitoneally with vehicle or aspirin $(20 \mathrm{mg} / \mathrm{kg}) 1$ hour prior to platelet activation with collagen $(50 \mu \mathrm{g} / \mathrm{kg})$.

\section{Histology}

Balb/c mice were injected intravenously with collagen (50 $\mu \mathrm{g} / \mathrm{kg}$ ) through the tail vein as described for the blood microsampling technique. At a time point corresponding to the maximal response ( 1 minute), lungs were immediately excised and frozen with liquid nitrogen. Sections of lung (10 $\mu \mathrm{m})$ were stained for the platelet-specific surface antigen CD41 ( $\alpha$ IIb) using a specific rat antimouse monoclonal immunoglobulin G (IgG) antibody (Abcam ab33661, Cambridge, United Kingdom).

\section{Data Analysis and Statistics}

The data obtained with the platelet monitoring technique were expressed as percentage increase in maximal radioactive counts from the baseline recording. All data were expressed as mean \pm SEM (standard error of the mean). Where statistical comparisons were made, a student's paired $t$-test or one-way average analysis of variance (ANOVA; repeated measurements) with Bonferroni post hoc multiple comparison test was used to compare mean values. Data were considered statistically significant with $p$-values $<0.05$.

The labelling efficiency of indium oxine and indium tropolone was calculated as the percentage of radioactive platelet counts obtained following removal of extracellular isotope relative to the counts obtained upon initial addition of radioactivity to platelet suspensions.

\section{Results}

\section{Indium Tropolone Efficiently Radiolabelled Isolated Mouse Platelets}

Indium oxine and indium tropolone radiolabelled isolated mouse platelets with an efficiency of 64.4 and $77.8 \%$, respectively (-Table 2). With both radiolabelling methods, the injection of sub-lethal doses of collagen $(50 \mu \mathrm{g} / \mathrm{kg})$ caused a rapid increase of platelet-associated radioactivity in the pulmonary vasculature, which reached a maximal level after approximately 1 minute before returning to basal levels within 10 minutes (-Fig. 1).

\section{Platelet Thromboembolism Caused a Measurable Fall in Circulating Platelets}

The radiolabelled platelet aggregation response (-Fig. 1) has previously been shown to be accompanied by a fall in radioactive counts in areas external to the lung, which then return to basal levels. ${ }^{8}$ Prior to devising microsampling techniques, we conducted proof of principle studies to confirm that platelet accumulation in the lung led to quantifiable reductions in 
Table 2 Efficiency of indium oxine and indium tropolone in radiolabelling mouse platelets

\begin{tabular}{|l|l|}
\hline \multicolumn{2}{|l|}{ Indium oxine } \\
\hline Counts upon initial addition of ${ }^{111} \mathrm{In}$ & $102,905 \pm 4,271$ \\
\hline Counts following platelet labelling with ${ }^{111} \mathrm{In}$ & $66,158 \pm 2,165$ \\
\hline \% radioactive labelling & $64.4 \pm 0.0$ \\
\hline Indium tropolone & $69,333 \pm 3,232$ \\
\hline Counts upon initial addition of ${ }^{111} \mathrm{In}$ & $55,000 \pm 2,733$ \\
\hline Counts following platelet labelling with ${ }^{111} \mathrm{In}$ & $77.8 \pm 3.8$ \\
\hline$\%$ radioactive labelling &
\end{tabular}

Notes: Indium oxine and indium tropolone radiolabelled mouse platelets with an efficiency of 64.4 and $77.8 \%$, respectively. Efficiency was calculated as percentage of radioactive counts following platelet labelling procedures compared with radioactive counts upon initial addition of indium oxine or indium tropolone.

circulating platelet counts by collecting blood by cardiac puncture in anaesthetized mice 1 minute after platelet activation with collagen $(12.5-50 \mu \mathrm{g} / \mathrm{kg})$. Collagen at 25 and $50 \mu \mathrm{g} / \mathrm{kg}$ caused a significant drop in platelet counts in blood samples compared with saline control (-Fig. 2). We therefore proceeded to refine this technique using microsampling techniques at multiple time points.

\section{Changes in Circulating Platelets were Measurable Using Microsampling}

Blood microsamples were collected before and after intravenous injection of collagen ( $50 \mu \mathrm{g} / \mathrm{kg}$ ), thrombin (65 IU/kg)

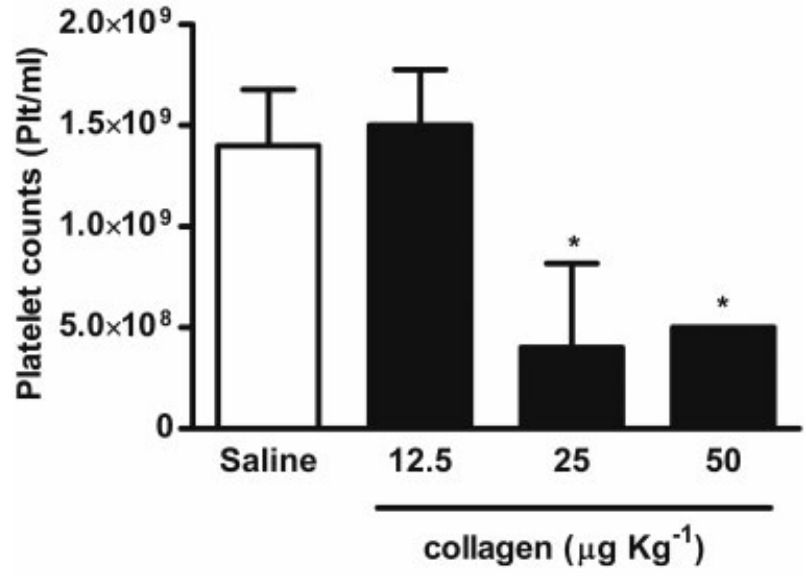

Fig. 2 Measurement of circulating platelet counts following collagen injection using cardiac puncture. Anaesthetized mice were injected intravenously (femoral vein) with increasing doses of collagen $(12.5-50 \mu \mathrm{g} / \mathrm{kg}$ ) or PBS (phosphate-buffered saline). After 1 minute, blood was collected by cardiac puncture and incubated with Stromatol. Collagen at 25 and $50 \mu \mathrm{g} /$ $\mathrm{kg}$ caused a significant reduction in platelet counts, 57 and 64\%, respectively, compared with saline control. One-way ANOVA (analysis of variance) with Bonferroni post hoc test; $n=4\left({ }^{*} p<0.05\right)$.

or $\operatorname{ADP}(400 \mu \mathrm{g} / \mathrm{kg})$. The data show a significant reduction in platelet counts 1 minute after injection of collagen ( - Fig. 3A; ${ }^{* *} p<0.01$ ) or thrombin ( $\mathbf{F i g . ~ 3 B ; ~}{ }^{*} p<0.05$ ) compared with basal platelet counts. After 10 minutes, the counts were restored for both agonists so that the platelet counts at 10 minutes were not significantly different to basal counts ( $p>0.05$; - Fig. 3A, B). These results were validated by
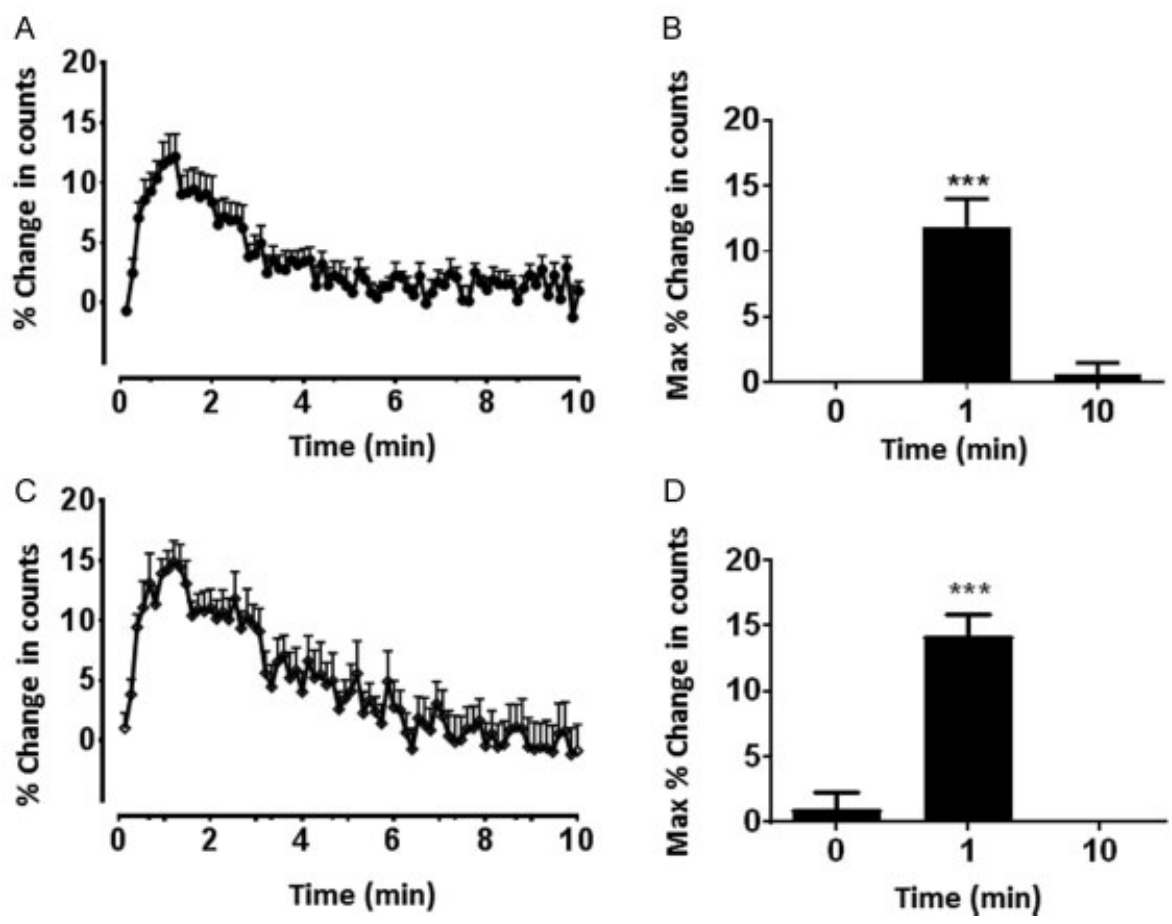

Fig. 1 Comparison of indium oxine and indium tropolone in a radiolabelled thromboembolic model of platelet thromboembolism in vivo. Mouse platelets were isolated, radiolabelled with $1.8 \mathrm{MBq}$ of indium oxine (A, B) or indium tropolone (C, D) and injected intravenously into a donor mouse. A thromboembolic response was induced via the intravenous injection of collagen and the changes in platelet-associated counts in the pulmonary vasculature were measured over 10 minutes. Platelet counts rapidly increased and reached a peak 1 minute after collagen injection and returned to baseline after 10 minutes. Repeated measures one-way ANOVA (analysis of variance) with Bonferroni post hoc test; $n=5\left({ }^{* * *} p<0.0001\right)$. 

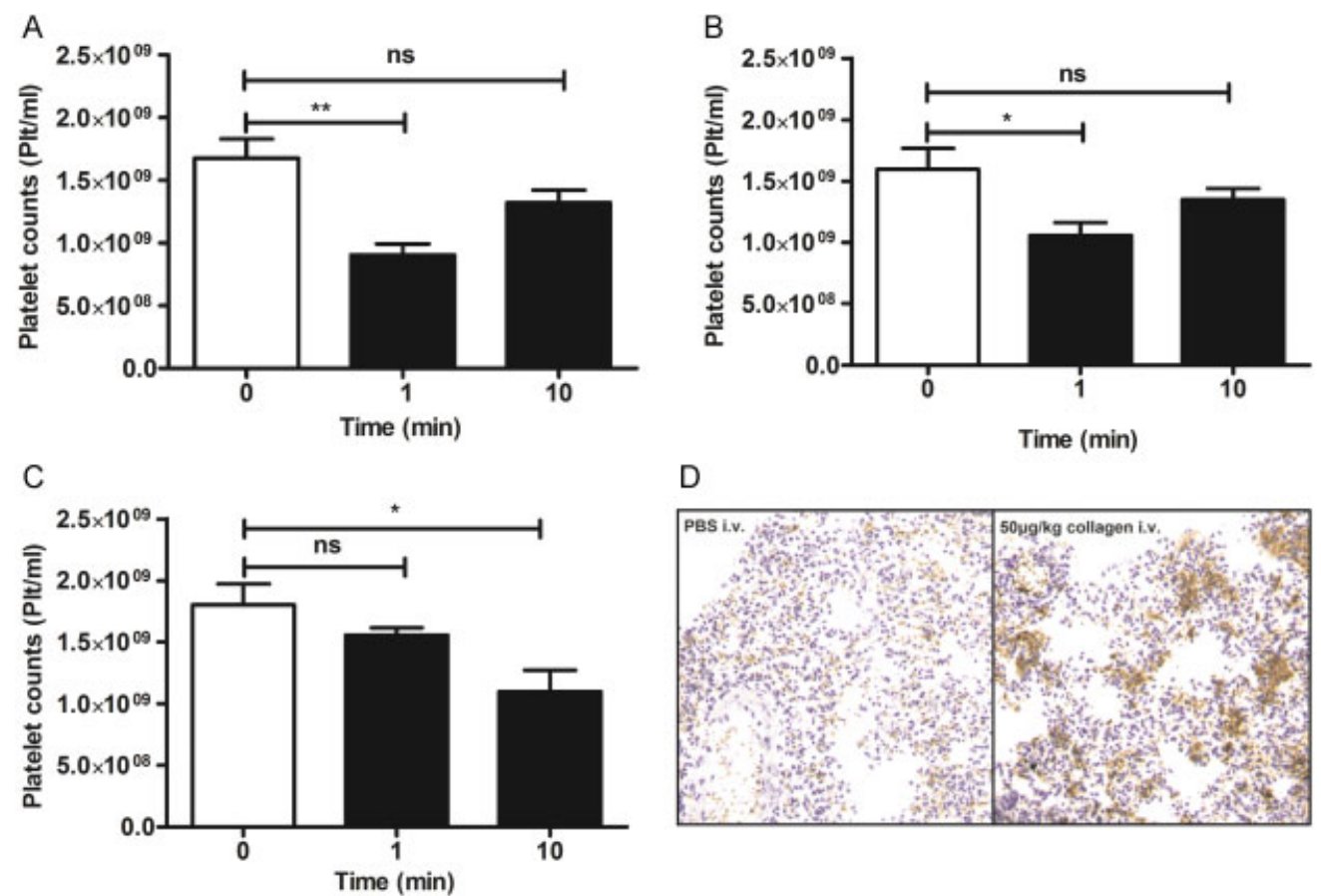

Fig. 3 Measurement of circulating platelet counts following agonist administration using blood microsampling. (A) Anaesthetized mice were intravenously injected with collagen $(50 \mu \mathrm{g} / \mathrm{Kg}$ ), thrombin $(65 \mathrm{lU} / \mathrm{kg})$, adenosine diphosphate (ADP; $400 \mu \mathrm{g} / \mathrm{kg})$ or saline. Blood $(2 \mu \mathrm{L})$ was collected prior to injection and 1 and 10 minutes after injection by microsampling and platelet counts determined. After 1 minute, both (A) collagen $\left(n=5 ;{ }^{* *} p<0.01\right)$ and (B) thrombin $\left(n=4 ;{ }^{*} p<0.05\right)$, caused a significant reduction in platelet counts, whereas (C) ADP had no effect at 1 minute although a significant reduction in counts was observed at 10 minutes. Platelet counts had recovered 10 minutes after injection of both collagen and thrombin. Repeated measures one-way ANOVA (analysis of variance) with Bonferroni post hoc test. (D) Sections of mouse lung tissue viewed at $\times 20$ magnification stained for platelet CD41 following injection of saline or collagen showed the formation of aggregates following collagen administration.

immunohistochemistry where collagen caused the accumulation of platelet aggregates, visible as brown staining at $\times 20$ magnification, in lung tissue; however, this did not occur with the PBS (phosphate-buffered saline) control (- Fig. 3D). In contrast, platelet activation with ADP did not show a significant drop in platelet counts after 1 minute (23.9\%; $p>0.05)$, but after 10 minutes a significant fall in platelet counts was detected (37.9\%; ${ }^{*}<0.05$; - Fig. $\left.3 \mathbf{3}\right)$.

\section{Aspirin-Inhibited Thromboembolism-Induced Reductions in Circulating Platelets}

Aspirin $(20 \mathrm{mg} / \mathrm{kg})$ significantly inhibited the reduction in platelet counts from 38.8 to $23.4 \%$ compared with vehicle control following platelet activation with collagen $50 \mu \mathrm{g} / \mathrm{kg}$ (-Fig. 4).

\section{Discussion}

Our group has previously developed a now well-established mouse model of radiolabelled platelet thromboembolism. The radioisotope required, indium oxine, is increasingly difficult to source, which led us to develop a new approach for radiolabelling mouse platelets. Here, we have demonstrated indium tropolone to be an effective alternative method to radiolabel mouse platelets, which is shown to radiolabel platelets more efficiently (-Table 2 ). With both radiolabelling approaches, we observed changes in radioactive counts following agonist

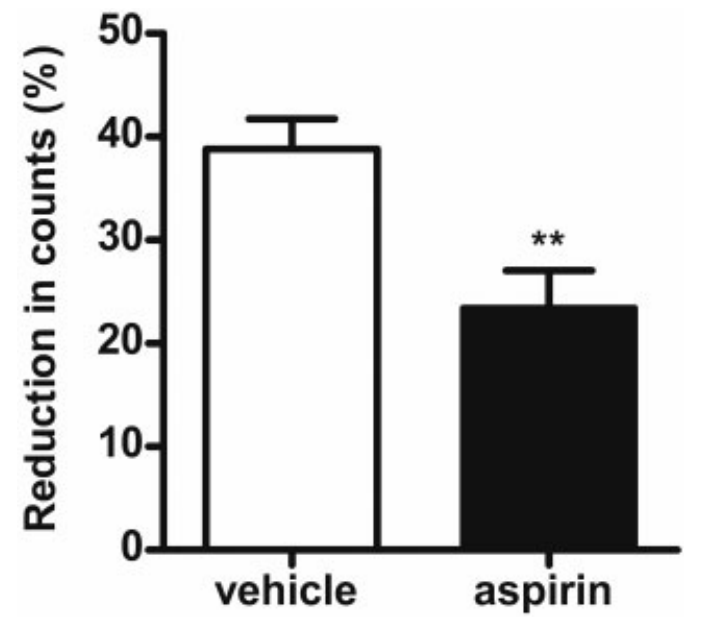

Fig. 4 Measurement of the effect of aspirin on circulating platelet counts following collagen injection using blood microsampling. Mice were pre-treated for 1 hour with aspirin $(20 \mathrm{mg} / \mathrm{kg})$ or vehicle $(n=6$ per group) and then intravenously injected with collagen $(50 \mu \mathrm{g} / \mathrm{kg})$ or saline under general anaesthesia. Blood $(2 \mu \mathrm{L})$ was collected before and 1 minute after collagen injection by microsampling and platelet counts determined. Platelet counts dropped significantly in the vehicle control group 1 minute after collagen injection, while treatment with aspirin significantly inhibited this reduction $(n=5)$. Unpaired one-tailed $t$-test $\left({ }^{* *} p<0.01\right)$. 
injection that have been previously shown to correlate with the formation and consequent resolution of thromboemboli in the dense pulmonary vascular bed. ${ }^{8}$ In the present study, we did not measure platelet counts in other organs or regions. Previously, however, we have shown platelet accumulation in the pulmonary vasculature to coincide with a concomitant fall in peripheral regions. ${ }^{8}$ This specific accumulation of platelets in the pulmonary vascular bed combined with histological analysis of lung sections and inhibition of the response by antithrombotic therapy has led us to conclude previously that our model is driven by platelet aggregation rather than, for example, adhesion to the vasculature. ${ }^{8}$

Platelet monitoring has the advantage of measuring platelet aggregation in real time and in situ in the presence of an intact vascular endothelium. This contrasts with established vascular injury model in which the vascular endothelium is damaged to induce thrombus formation ${ }^{16}$ and which do not functionally isolate the platelet response. ${ }^{3}$ Our model has been particularly useful in determining the influence of vascular mediators such as $\mathrm{NO}$ in regulating platelets in vivo, an issue that produced contradictory findings in vascular injury studies. ${ }^{17-21}$ Real-time monitoring does, however, require specific skills, resources and facilities. These issues have proven to be barriers to uptake. We therefore developed blood microsampling as a refined mouse model to measure platelet aggregation in vivo. Based on published evidence that platelet activation with collagen causes platelets to accumulate in the lung with a consequent drop in circulating platelet-associated radioactive counts, ${ }^{8}$ blood samples were collected at specific time points following platelet activation and platelet counts measured. Blood sampling via cardiac puncture was performed under terminal anaesthesia as proof of principle and confirmed a measurable and significant reduction in circulating platelet counts in blood samples collected 1 minute after collagen injection (-Fig. 2), which correlates with the maximal increase in platelet-associated radioactive counts detected in the pulmonary vasculature using radiolabelled platelet monitoring (-Fig. 1). We then refined this approach by developing a blood microsampling method where multiple small incisions were performed on the tail vein. Similarly to the cardiac puncture sampling approach, collagen and thrombin, but not ADP, caused a significant reduction in platelet counts compared with the saline control group which were then restored over a 10-minute period (-Fig. 3A). These results were validated by immunohistochemistry where platelet activation with collagen caused the formation of platelet aggregates in the mouse lung tissue, whereas this did not occur following saline challenge ( - Fig. 3B). We conclude therefore that the reported reductions in circulating platelets were due to accumulation of platelet aggregation in the pulmonary vascular bed.

To validate that reductions in platelet counts were driven by platelet activation, we used the established antiplatelet drug aspirin. Aspirin inhibits the process of platelet activation by inhibiting the generation of pro-aggregatory thromboxane $A_{2}$ from cyclooxygenase. The significant effect of aspirin on collagen-mediated changes in platelet counts links this observation with platelet activation and suggests that the method may be used to determine the impact of novel antiplatelet compounds or to investigate other pharmacological or genetic interventions hypothesized to impact the platelet activation process.

Based on the results presented in this paper, blood microsampling during platelet thromboembolism represents a refined and non-invasive alternative to thromboembolic mortality models which avoids pain and suffering in experimental mice and allows the acquisition of a higher number of samples from each mouse, leading to a considerable reduction of animal numbers and therefore also of costs. Microsampling does not provide the continuous data acquisition that arises from real-time monitoring of radiolabelled platelet accumulation so that the latter remains the gold standard in vivo model. The importance of real-time monitoring of radiolabelled platelet accumulation is highlighted by our inability to measure a significant ADP response with microsampling within 1 minute. Microsampling could, however, be implemented by those groups without access to the expertise or facilities for real-time monitoring and where the full-time course of the response is not required.

Thromboembolic mortality models employ survival or limb paralysis as endpoints, thus requiring up to 15 to 20 animals per group to adequately power studies. The $3 R$ s principles, now a requirement under EU legislation, require that animals are replaced with non-animal models wherever possible (replacement), that the number of animals is reduced to the minimum needed to obtain the results sought (reduction) and that, when used, animal procedures are refined to minimize their suffering and, thus, that the lowest severity levels are used (refinement). In compliance with the current EU directive, thromboembolic mortality models could be replaced with refined real-time monitoring or, where this is not feasible, microsampling approaches to comply with legislation relating to the 3Rs. Indeed, the application of our platelet monitoring technique has already been shown to reduce animal numbers by up to $85 \%^{7}$ and, moreover, the refined microsampling model presented here was able to further reduce these numbers by around $50 \%$. This additional reduction is achieved by the need for donor mice in monitoring studies for terminal bleeding to obtain platelets for radiolabelling and subsequent infusion into recipient mice. As with real-time platelet monitoring, blood microsampling procedures are conducted at lower severity levels as animals are kept under terminal anaesthesia throughout procedures and their suffering would be minimized to constitute a refinement. This contrasts sharply with thromboembolic mortality studies conducted without anaesthesia or analgesia in which animals are subjected to considerable pain and suffering.

An additional advantage of both the real-time monitoring and microsampling techniques reported here is that effects caused by pharmacological or genetic manipulation can be directly attributed to platelets rather than the less specific endpoint of death or paralysis that is used in mortality studies. Fewer subsequent experiments are therefore likely to be required to demonstrate a platelet-mediated effect. In addition, by using sub-lethal doses of platelet agonists in 
microsampling studies, we suggest that greater sensitivity will be achieved compared with mortality studies since effects are tested at submaximal agonist doses.

In conclusion, we report a simplistic method for assessing platelet thromboembolism via microsampling. This method provides an alternative to thromboembolic mortality approaches where the gold standard model, real-time monitoring, is not feasible allowing scientists to demonstrate compliance with EU legislation concerning the 3Rs.

\section{What is known about this topic?}

- Mouse models of thromboembolic mortality are used worldwide and inflict considerable suffering and pain. According to European Union (EU) legislation, animal procedures must be conducted in the context of replacement, refinement and reduction.

- Platelet monitoring was developed as a refined mouse model of thromboembolism and measures real-time increases in radioactive-associated platelet counts in the pulmonary vasculature following the induction of a thromboembolism. This is associated with a fall in platelet counts in the peripheral vasculature.

- Real-time platelet monitoring represents a refinement of mortality models since procedures are conducted under general anaesthesia and considerable reductions in animal numbers are achieved. However, the technique requires work with radiation, suitable facilities and particular training.

\section{What does this Paper add?}

- We developed a refined mouse model of thromboembolism based on blood microsampling where the fall in circulating platelet counts during thromboembolism was measured and shown to be associated with the accumulation of platelets in the pulmonary vasculature. This procedure is conducted under general anaesthesia and allows the collection of three samples from a single mouse.

- Blood microsampling drastically reduces animal numbers and delivers data by using a simplistic procedure that does not require work with radiation and that can be easily implemented by research groups currently using mortality models.

- An alternative procedure to radiolabel mouse platelets was developed whereby indium tropolone was used as opposed to indium oxine since the latter is no longer commercially available in the United Kingdom.

\section{Grants}

This work was supported by a Research Grant from the National Centre for the Replacement, Refinement and Reduction of Animals in Research (NC/M000079/1).

\section{References}

1 Gawaz M. Role of platelets in coronary thrombosis and reperfusion of ischemic myocardium. Cardiovasc Res 2004;61(03): 498-511

2 Russell JC, Proctor SD. Small animal models of cardiovascular disease: tools for the study of the roles of metabolic syndrome, dyslipidemia, and atherosclerosis. Cardiovasc Pathol 2006;15(06):318-330

3 Nieswandt B, Aktas B, Moers A, Sachs UJ. Platelets in atherothrombosis: lessons from mouse models. J Thromb Haemost 2005;3(08):1725-1736

4 Mizurini DM, Francischetti IM, Monteiro RQ. Aegyptin inhibits collagen-induced coagulation activation in vitro and thromboembolism in vivo. Biochem Biophys Res Commun 2013;436(02): 235-239

5 Momi S, Caracchini R, Falcinelli E, Evangelista S, Gresele P. Stimulation of platelet nitric oxide production by nebivolol prevents thrombosis. Arterioscler Thromb Vasc Biol 2014;34(04): $820-829$

6 Ma D, Mizurini DM, Assumpção TC, et al. Desmolaris, a novel factor XIa anticoagulant from the salivary gland of the vampire bat (Desmodus rotundus) inhibits inflammation and thrombosis in vivo. Blood 2013;122(25):4094-4106

7 Emerson M. Refinement, reduction and replacement approaches to in vivo cardiovascular research. Br J Pharmacol 2010; 161(04):749-754

8 Tymvios C, Jones S, Moore C, Pitchford SC, Page CP, Emerson M. Real-time measurement of non-lethal platelet thromboembolic responses in the anaesthetized mouse. Thromb Haemost 2008; 99(02):435-440

9 May GR, Herd CM, Butler KD, Page CP. Radioisotopic model for investigating thromboembolism in the rabbit. J Pharmacol Methods 1990;24(01):19-35

10 Solomon A, Smyth E, Mitha N, et al. Induction of platelet aggregation after a direct physical interaction with diesel exhaust particles. J Thromb Haemost 2013;11(02):325-334

11 Smyth E, Solomon A, Vydyanath A, et al. Induction and enhancement of platelet aggregation in vitro and in vivo by model polystyrene nanoparticles. Nanotoxicology 2015;9(03):356-364

12 Apostoli GL, Solomon A, Smallwood MJ, Winyard PG, Emerson M. Role of inorganic nitrate and nitrite in driving nitric oxide-cGMPmediated inhibition of platelet aggregation in vitro and in vivo. J Thromb Haemost 2014;12(11):1880-1889

13 Emerson M, Momi S, Paul W, Alberti PF, Page C, Gresele P. Endogenous nitric oxide acts as a natural antithrombotic agent in vivo by inhibiting platelet aggregation in the pulmonary vasculature. Thromb Haemost 1999;81(06):961-966

14 Tymvios C, Moore C, Jones S, Solomon A, Sanz-Rosa D, Emerson M. Platelet aggregation responses are critically regulated in vivo by endogenous nitric oxide but not by endothelial nitric oxide synthase. Br J Pharmacol 2009;158(07):1735-1742

15 Kilkenny C, Browne WJ, Cuthill IC, Emerson M, Altman DG. Improving bioscience research reporting: the ARRIVE guidelines for reporting animal research. PLoS Biol 2010;8(06): e1000412

16 Bodary PF, Eitzman DT. Animal models of thrombosis. Curr Opin Hematol 2009;16(05):342-346

17 Heeringa P, van Goor H, Itoh-Lindstrom Y, et al. Lack of endothelial nitric oxide synthase aggravates murine accelerated anti-glomerular basement membrane glomerulonephritis. Am J Pathol 2000; 156(03):879-888

18 Iafrati MD, Vitseva O, Tanriverdi K, et al. Compensatory mechanisms influence hemostasis in setting of eNOS deficiency. Am J Physiol Heart Circ Physiol 2005;288(04):H1627-H1632

19 Marjanovic JA, Li Z, Stojanovic A, Du X. Stimulatory roles of nitricoxide synthase 3 and guanylyl cyclase in platelet activation. J Biol Chem 2005;280(45):37430-37438

20 Ozüyaman B, Gödecke A, Küsters S, Kirchhoff E, Scharf RE, Schrader J. Endothelial nitric oxide synthase plays a minor role 
in inhibition of arterial thrombus formation. Thromb Haemost 2005;93(06):1161-1167

21 Dayal S, Wilson KM, Leo L, Arning E, Bottiglieri T, Lentz SR. Enhanced susceptibility to arterial thrombosis in a murine model of hyperhomocysteinemia. Blood 2006;108(07):2237-2243

22 Assafim M, Frattani FS, Ferreira MS, Silva DM, Monteiro RQ Zingali RB. Exploiting the antithrombotic effect of the (pro) thrombin inhibitor bothrojaracin. Toxicon 2016;119:46-51

23 Choi JH, Kim SJ, Kim S. A novel anticoagulant protein with antithrombotic properties from the mosquito Culex pipiens pallens. Int J Biol Macromol 2016;93:156-166

24 Huang SW, Kuo HL, Hsu MT, Tseng YJ, Lin SW, Kuo SC, Peng HC, Lien JC, Huang TF. A novel thromboxane receptor antagonist, nstpbp5185, inhibits platelet aggregation and thrombus formation in animal models. Thrombosis and haemostasis 2016; 116:285-99

25 Kim JH, Lee J, Kang S, Moon H, Chung KH, Kim KR. Antiplatelet and Antithrombotic Effects of the Extract of Lindera obtusiloba Leaves. Biomol Ther (Seoul) 2016;24:659-664
26 Park J, Lee B, Choi H, Kim W, Kim HJ, Cheong H. Antithrombosis activity of protocatechuic and shikimic acids from functional plant Pinus densiflora Sieb. et Zucc needles. J Nat Med 2016; 70:492-501

27 Misra A, Anil Kumar KS, Jain M, Bajaj K, Shandilya S, Srivastava S, Shukla P, Barthwal MK, Dikshit M, Dikshit DK. Synthesis and evaluation of dual antiplatelet activity of bispidine derivatives of N-substituted pyroglutamic acids. Eur J Med Chem 2016;110:1-12

28 Saito MS, Lourenco AL, Dias LR, Freitas AC, Vitorino MI, Albuquerque MG, Rodrigues CR, Cabral LM, Dias EP, Castro HC, Satlher PC. Antiplatelet pyrazolopyridines derivatives: pharmacological, biochemical and toxicological characterization. J Enzyme Inhib Med Chem 2016;31:1591-601

29 Lee W, Lee J, Kulkarni R, Kim MA, Hwang JS, Na M, Bae JS. Antithrombotic and antiplatelet activities of small-molecule alkaloids from Scolopendra subspinipes mutilans. Sci Rep 2016;6:21956

$30 \mathrm{Kim}$ TH, Lee KM, Hong ND, Jung YS. Anti-platelet and antithrombotic effect of a traditional herbal medicine Kyung-OkKo. J Ethnopharmacol 2016;178:172-9 